\title{
Galerkin Methods for Singular Integral Equations
}

\author{
By K. S. Thomas
}

\begin{abstract}
The approximate solution of a singular integral equation by Galerkin's method is studied. We discuss the theoretical aspects of such problems and give error bounds for the approximate solution.
\end{abstract}

1. Introduction. In this paper we will discuss Galerkin's method for the approximate solution of the singular integral equation

$$
u(s)-\frac{1}{\pi} \int_{-1}^{+1} \frac{k(s, t) u(t)}{t-s} d t=f(s),
$$

in which $k(s, t)$ is a real-valued kernel (assumed smooth), $f(s)$ is a given function, and $u(s)$ is the unknown function. The integral is to be interpreted as a Cauchy principal value throughout the paper. It is well known that the solution of (1.1) is not unique unless one restricts the space of functions in which the solution is sought in some manner. In this paper we restrict $u$ to lie in $L^{2}[-1,1]$.

In Section 2 we outline the theory for singular integral equations. This is mainly based on the treatment in [12]. We will show the uniqueness of the $L^{2}$-solution of (1.1). Also, from the theoretical treatment, one obtains the asymptotic behavior of the solution at \pm 1 . The solution, in fact, nearly always has endpoint singularities.

In Section 3 we give the error analysis for Galerkin's method. The main result is that

$$
\left\|u-u_{n}\right\|_{L^{2}} \leqslant C(1+o(1))\left\|\left(I-P_{n}\right) u\right\|,
$$

where $u_{n}$ is the approximate solution, $C$ a constant, and $P_{n}$ is the projection operator from $L^{2}[-1,1]$ into the space of trial functions.

Section 4 deals with the use of spline functions as a basis for Galerkin's method. The endpoint singularities mean that one must use splines on nonuniform partitions and singular functions as the trial space. We show how to calculate a partition that gives an asymptotic rate of convergence of $O\left(N^{-k}\right)$ where $k-1$ is the degree of the spline. The paper is concluded by a numerical example.

The basis for this paper is the section on singular integral equations in the book by Cherruault [4]. Different treatments for singular integral equations include [6], [7], [8], [9], [10].

2. Theoretical Treatment of Singular Integral Equations. The theoretical analysis of singular integral equations using complex variable theory dates from Carleman [3] and may be found in numerous text books (e.g. Tricomi [15], Muskhelishvili [12]). We follow the treatment of [12, Chapters 10,14] and show that (1.1) has a

Received May 23, 1977; revised May 21, 1980.

AMS (MOS) subject classifications (1970). Primary 65R05.

(C) 1981 American Mathematical Society 0025-5718/81/0000-0015/\$04.25 
unique solution on $L^{2}[-1,1]$. The reader is left to fill in the details.

[12] shows that, in order to solve a singular integral equation such as (1.1) on an open interval, one must specify the class $C$ of functions in which the solution is sought. This is often accomplished by imposing additional boundary conditions on the solution. The questions of existence and uniqueness of solutions are answered by computing the index $\kappa$ (an integer) of the integral equation with respect to the class of functions $\mathcal{C}$. (When considering singular integral equations on a contour in the complex plane the index does not depend on additional boundary conditions.)

The integral equation (1.1) is rewritten in the form

$$
A u-K u=f
$$

where

$$
A u(s)=u(s)-\frac{k(s, s)}{\pi} \int_{-1}^{+1} \frac{u(t)}{t-s} d t
$$

and

$$
K u(s)=\frac{1}{\pi} \int_{-1}^{+1} \frac{k(s, t)-k(s, s)}{t-s} u(t) d t .
$$

The operator $A$ is called the dominant part of the integral equation. $K$ is compact because of the smoothness (Hölder-continuity) of $k(s, t)$.

The first stage of the theoretical analysis is to solve the equation

$$
A v=g \text {, }
$$

where $v, g \in L^{2}[-1,1]$.

[12, Chapter 14] is used to determine the analytic solution of (2.4). We define the function

$$
\theta(s)=\frac{1}{\pi} \underset{(-\pi / 2, \pi / 2)}{\arctan } k(s, s)
$$

integers $n_{1}, n_{2}$ such that

$$
-1<\theta(1)+n_{1}<1, \quad-1<-\theta(-1)+n_{2}<1
$$

and the function

$$
\Omega(s)=(1-s)^{n_{1}}(1+s)^{n_{2}} \exp \left[\int_{-1}^{+1} \frac{\theta(t)}{t-s} d t\right] .
$$

The index of (2.4) is then given by

$$
\kappa=-\left(n_{1}+n_{2}\right)
$$

and the analytic solution of (2.4) by

$$
\begin{aligned}
v= & K^{*} g \\
= & \frac{g(s)}{1+k^{2}(s, s)}+\frac{k(s, s) \Omega(s)}{\pi \sqrt{1+k^{2}(s, s)}} \int_{-1}^{+1} \frac{g(t)}{\sqrt{\left(1+k^{2}(t, t)\right) \Omega(t)(t-s)}} d t \\
& +\frac{k(s, s) \Omega(s) P_{\kappa-1}(s)}{\sqrt{1+k^{2}(s, s)}}
\end{aligned}
$$

where $P_{\kappa-1}(s)$ is an arbitrary polynomial of degree $\kappa-1,\left(P_{\kappa-1}(s) \equiv 0\right.$ if $\left.\kappa<0\right)$.

The analytic solution gives useful information about the asymptotic behavior near the ends $s= \pm 1$. By applying the results in [12, Chapter 4] we can show that 


$$
\begin{array}{ll}
v(s) \sim a_{1}(1-s)^{\theta(1)+n_{1}}, & \text { near } s=1, \\
v(s) \sim a_{2}(1+s)^{-\theta(-1)+n_{2}}, & \text { near } s=-1,
\end{array}
$$

provided $\theta(1) \neq 0$ and $\theta(-1) \neq 0$.

If $\theta(s)$ has a zero of order $r$ at $s= \pm 1$, then

$$
v(s) \sim f(s)+b_{1}(1 \pm s)^{r} \log (1 \pm s), \quad \text { near } s= \pm 1 .
$$

The conditions (2.6) indicate that $n_{1}, n_{2}$ are only determined to within \pm 1 . Only by imposing the condition $v \in \mathcal{C}$ do we determine $n_{1}, n_{2}$. By choosing $n_{1}, n_{2}$ to minimize the value $n_{1}+n_{2}$ can take without violating the condition $v \in \mathcal{C},(2.8)$ will give the most general solution in the class $\mathcal{C}$ and (2.7) the index.

If we require that $v \in L^{2}[-1,1]$, we should replace (2.6) by

$$
-\frac{1}{2}<\theta(1)+n_{1}<1, \quad-\frac{1}{2}<-\theta(-1)+n_{2}<1
$$

to ensure that our solution is square-integrable. We can manipulate the first of these inequalities to get

$$
-1 \leqslant-\frac{1}{2}-\theta(1)<n_{1}<1-\theta(1) \leqslant \frac{3}{2}
$$

since $-\frac{1}{2}<\theta(s)<\frac{1}{2}$. Hence, $n_{1}$ can only take the values 0 or 1 . Similarly $n_{2}$ can only take the values 0 or 1 . Hence, the minimum value of $n_{1}+n_{2}$, subject to $v \in L^{2}[-1,1]$, is zero, i.e. $\kappa=0$.

This establishes the uniqueness of the solution of the dominant equation.

Having solved the dominant part of the singular equation, the full equation can be reduced to the Fredholm equation,

$$
u-K^{*} K u=K^{*} f
$$

Since the operator $K^{*} K$ is compact, the usual theory of the Fredholm alternative is applicable. This means that either the integral equation will have a unique solution in $L^{2}[-1,1]$ or unity will be an eigenvalue of $K^{*} K$. We will assume for the remainder of the paper the existence of a unique $L^{2}$ solution.

Moreover, we have assumed that $f(s)$ and $k(s, t)$ are smooth functions, so that $g=K u+f$ defines a smooth function $g$. Since $u=K^{*} g, u(s)$ will have similar asymptotic behavior as $v(s)$ near $s= \pm 1$, governed by the equations (2.9)-(2.11).

3. The Error Analysis of Galerkin's Method. To give an error analysis of Galerkin's method, we rewrite (1.1) in the form

$$
u-K_{1} u-K_{2} u=f
$$

where

$$
K_{1} u(s)=\frac{1}{2 \pi} \int_{-1}^{+1} \frac{k(s, t)+k(t, s)}{t-s} u(t) d t
$$

and

$$
K_{2} u(s)=\frac{1}{2 \pi} \int_{-1}^{+1} \frac{k(s, t)-k(t, s)}{t-s} u(t) d t .
$$

The operator $K_{1}$ is skew-hermitian and the operator $K_{2}$ is symmetric and compact. We first give the analysis of Galerkin's method for the problem 


$$
B u=f,
$$

where $B=I-K_{1}$. We define the bilinear functional $a(u, v)=(B u, v)$ for $u, v \in$ $L^{2}[-1,1]$.

LEMMA 3.1. (i) $\operatorname{Re} a(u, u)=\|u\|^{2}, u \in L^{2}[-1,1]$.

(ii) $|a(u, v)| \leqslant\|B\|\|u\|\|v\|, u, v \in L^{2}[-1,1]$.

(iii) $B^{-1}$ exists and $\left\|B^{-1}\right\| \leqslant 1$.

Proof. (i) $\operatorname{Re}\left(K_{1} u, u\right)=\frac{1}{2}\left[\left(K_{1} u, u\right)+\overline{\left(K_{1} u, u\right)}\right]=\frac{1}{2}\left(\left(K_{1}+K_{1}^{*}\right) u, u\right)=0$ since $K_{1}^{*}$ $=-K_{1}$. Hence, $\operatorname{Re} a(u, u)=(u, u)-\operatorname{Re}\left(K_{1} u, u\right)=\|u\|^{2}$.

(ii) This follows directly from the Schwarz inequality.

(iii) From (i) and the Schwarz inequality, one can show $\|u\|^{2}<\|B u\|\|u\|$. Hence, $\|u\| \leqslant\|B u\|$. (If $\|u\| \neq 0$, the division is permissible, if $u=0$, the result is trivial.) This establishes the existence of $B^{-1}$, and $\left\|B^{-1}\right\| \leqslant 1$ is established from a standard result in functional analysis.

We let $S_{n} \subset L^{2}[-1,1]$ be the finite-dimensional subspace (the dimension of $S_{n}$ is $n$ ) in which we find the Galerkin approximation $u_{n}$. We let $P_{n}$ be the projection operator onto the subspace $S_{n}$. The Galerkin approximation will satisfy

$$
a\left(u_{n}, v_{n}\right)=\left(f, v_{n}\right) \text { for } v_{n} \in S_{n} \text {, }
$$

and the error in method is given by the following.

THEOREM 3.2. The error in Galerkin's method for the problem $B u=f$ is given by

$$
\left\|u-u_{n}\right\| \leqslant\|B\|\left\|\left(I-P_{n}\right) u\right\| .
$$

Proof. It is readily verified that $a\left(u-u_{n}, w_{n}\right)=0$ for all $w_{n} \in S_{n}$. Hence,

$$
\begin{aligned}
\left\|u-u_{n}\right\|^{2} & =\operatorname{Re} a\left(u-u_{n}, u-u_{n}\right) \leqslant\left|a\left(u-u_{n}, u-u_{n}\right)\right| \\
& \leqslant\left|a\left(u-u_{n}, u-P_{n} u\right)\right|+\left|a\left(u-u_{n}, P_{n} u-u_{n}\right)\right| \\
& =\left|a\left(u-u_{n},\left(I-P_{n}\right) u\right)\right| \leqslant\|B\|\left\|u-u_{n}\right\|\left\|\left(I-P_{n}\right) u\right\|
\end{aligned}
$$

from Lemma 3.1. Hence, we may divide through by $\left\|u-u_{n}\right\|$ and get the result.

For the full equation (3.1) we put

$$
B u=v .
$$

Then (3.1) is equivalent to

$$
v-K_{2} B^{-1} v=f
$$

Since $B^{-1}$ is continuous and $K_{2}$ compact, $K_{2} B^{-1}$ forms a compact operator. We can use the theory of prolongation and restriction operators developed in [11], [14] to produce error bounds. We define operators $q_{n}$ and $s_{n}$ as follows. $\phi_{i}(t), i=1, \ldots, n$, is a set of linearly independent functions that span $S_{n}$. Define

$$
q_{n}: E_{n} \rightarrow L^{2}[-1,1]
$$

by

$$
q_{n} \mathbf{v}_{n}=\sum_{j=1}^{n} v_{j} \phi_{j}
$$

and

$$
s_{n}: L^{2}[-1,1] \rightarrow E_{n}
$$


by

$$
s_{n} f=\left\{\left(f, \phi_{i}\right)\right\}
$$

It is readily confirmed that

$$
s_{n} q_{n}=G_{n}
$$

the Gramm matrix of the basis.

We put

$$
B_{n}=s_{n} B q_{n} \text {. }
$$

Then Galerkin's method for the problem (3.4) produces the linear equations

$$
B_{n} \mathbf{u}_{n}=s_{n} f
$$

and $q_{n} \mathbf{u}_{n}$ is the Galerkin approximation. However, [14, Eq. 4] is not satisfied by $q_{n}$, $s_{n}$, so we define prolongation and restriction operators $p_{n}, r_{n}$ by

$$
p_{n}=B q_{n}, \quad r_{n}=B_{n}^{-1} s_{n},
$$

and now $r_{n} p_{n}=I_{n}$.

We define our norm in $E_{n}$ by

$$
\left\|\mathbf{v}_{n}\right\|_{E_{n}}=\left\|q_{n} \mathbf{v}_{n}\right\|_{L^{2}}
$$

This norm is related to the Euclidean vector norm by the following

Lemma 3.3. (i) $\left(q_{n} \mathbf{f}_{n}, g\right)=\left\langle\mathbf{f}_{n}, s_{n} g\right\rangle, \mathbf{f}_{n} \in E_{n}, g \in L^{2}[-1,1]$ (i.e. $s_{n}^{*}=q_{n}, q_{n}^{*}=$ $\left.s_{n}\right)$.

(ii) $\left\|\mathbf{v}_{n}\right\|_{E_{n}}=\left\|G_{n}^{1 / 2} \mathbf{v}_{n}\right\|_{2}$, where $\left\langle\mathbf{f}_{n}, g_{n}\right\rangle=\sum f_{i} \bar{g}_{i}$, denotes the inner-product in $E_{n}$ and \|\|$_{2}$ is the usual Euclidean vector norm

$$
\left\|\mathbf{v}_{n}\right\|_{2}^{2}=\left\langle\mathbf{v}_{n}, \mathbf{v}_{n}\right\rangle \text {. }
$$

Proof.

$$
\begin{gathered}
\left(q_{n} \mathbf{f}_{n}, g\right)=\left(\sum_{i=1}^{n} f_{i} \phi_{i}, g\right)=\sum_{i=1}^{n} f_{i}\left(\overline{g, \phi_{i}}\right)=\left\langle\mathbf{f}_{n}, s_{n} g\right\rangle . \\
\left\|\mathbf{v}_{n}\right\|_{E_{n}}^{2}=\left\|q_{n} \mathbf{v}_{n}\right\|_{L^{2}}^{2}=\left(q_{n} \mathbf{v}_{n}, q_{n} \mathbf{v}_{n}\right)=\left\langle\mathbf{v}_{n}, s_{n} q_{n} \mathbf{v}_{n}\right\rangle \\
=\left\langle\mathbf{v}_{n}, G_{n} \mathbf{v}_{n}\right\rangle=\left\langle G_{n}^{1 / 2} \mathbf{v}_{n}, G_{n}^{1 / 2} \mathbf{v}_{n}\right\rangle=\left\|G_{n}^{1 / 2} \mathbf{v}_{n}\right\|^{2} .
\end{gathered}
$$

Lemma 3.3 is useful in establishing the stability of the prolongation and restriction operators.

THEOREM 3.4. If the norm in Euclidean space is defined by (3.14), then (i) $\left\|p_{n}\right\| \leqslant\|B\|$, (ii) $\left\|r_{n}\right\| \leqslant 1$.

Proof. (i) If $\mathbf{u}_{n} \in E_{n}$ with $\left\|\mathbf{u}_{n}\right\|_{E_{n}}=1$,

$$
\left\|p_{n} \mathbf{u}_{n}\right\|_{L^{2}}=\left\|B q_{n} \mathbf{u}_{n}\right\|_{L^{2}} \leqslant\|B\|\left\|q_{n} u_{n}\right\|_{L^{2}}=\|B\| .
$$

(ii) Let $f \in L^{2}[-1,1]$ with $\|f\|=1$. Then

$$
\left\|r_{n} f\right\|_{E_{n}}=\left\|G_{n}^{1 / 2} r_{n} f\right\|_{2}=\left\|G_{n}^{1 / 2} B_{n}^{-1} s_{n} f\right\|_{2} .
$$

Now

$$
\begin{gathered}
B_{n}=G_{n}-s_{n} K_{1} q_{n}=G_{n}^{1 / 2}\left(I-G_{n}^{-1 / 2} s_{n} K_{1} q_{n} G_{n}^{-1 / 2}\right) G_{n}^{1 / 2} \\
\therefore \quad B_{n}^{-1}=G_{n}^{-1 / 2}\left(I-G_{n}^{-1 / 2} s_{n} K_{1} q_{n} G_{n}^{-1 / 2}\right)^{-1} G_{n}^{-1 / 2}
\end{gathered}
$$


So

$$
\left\|r_{n} f\right\|_{E_{n}} \leqslant\left\|\left(I-G_{n}^{-1 / 2} s_{n} K_{1} q_{n} G_{n}^{-1 / 2}\right)^{-1}\right\|_{2} \cdot\left\|G_{n}^{-1 / 2} s_{n} f\right\|_{2}<\left\|G_{n}^{-1 / 2} s_{n} f\right\|_{2},
$$

since $G_{n}^{-1 / 2} s_{n} K_{1} q_{n} G_{n}^{-1 / 2}$ is skew-symmetric (Lemma 3.3(i)) and thus

$$
\left\|\left(I-G_{n}^{-1 / 2} s_{n} K_{1} q_{n} G_{n}^{-1 / 2}\right)^{-1}\right\|_{2}<1 .
$$

Now

$$
\left\|G_{n}^{-1 / 2} s_{n} f\right\|_{2}^{2}=\left\langle s_{n} f, G_{n}^{-1} s_{n} f\right\rangle=\left(f, q_{n} G_{n}^{-1} s_{n} f\right) .
$$

$q_{n} G_{n}^{-1} s_{n}$ is simply the projection operator $P_{n}$ from $L^{2}[-1,1]$ into $S_{n}$ and thus $\left\|q_{n} G_{n}^{-1} s_{n}\right\|<1$. Hence, $\left(f, q_{n} G_{n}^{-1} S_{n} f\right)<\|f\|^{2}$

$$
\therefore\left\|r_{n} f\right\|_{E_{n}}<\|f\|
$$

establishing the result.

LEMMA 3.5. For $g \in L^{2}[-1,1]$ we have

$$
\left\|\left(1-p_{n} r_{n}\right) g\right\| \leqslant\|B\|^{2}\left\|\left(I-P_{n}\right) B^{-1} g\right\| .
$$

Proof.

$$
\begin{aligned}
\left\|\left(1-p_{n} r_{n}\right) g\right\| & =\left\|B\left(B^{-1}-q_{n} B_{n}^{-1} s_{n}\right) g\right\| \\
& \leqslant\|B\|\left\|\left(B^{-1}-q_{n} B_{n}^{-1} s_{n}\right) g\right\|<\|B\|^{2}\left\|\left(I-P_{n}\right) B^{-1} g\right\|,
\end{aligned}
$$

since the second term is merely the error in Galerkin's method applied to the problem $B W=g$ and we may use Theorem 3.2.

Following the method described in [14, Section 6], (3.4) is to be approximated by

$$
\left(I_{n}-r_{n} K_{2} B^{-1} p_{n}\right) \mathbf{v}_{n}=r_{n} f
$$

which is equivalent to

$$
\left(G_{n}-s_{n}\left(K_{1}+K_{2}\right)\right) \mathbf{v}_{n}=s_{n} f
$$

the Galerkin approximation of the full equation. For brevity we put

$$
K_{n}=r_{n} K_{2} B^{-1} p_{n} \text {. }
$$

[14, Theorem 3.2] can now be used to establish a bound on $\left\|\left(I_{n}-K_{n}\right)^{-1}\right\|_{E_{n}}$. We recall the assumption of a unique $L^{2}$-solution which implies the existence of $\left(I-K_{2} B^{-1}\right)^{-1}$.

THEOREM 3.6. If $\left(I-K_{2} B^{-1}\right)^{-1}$ exists and

$$
\delta_{n}=\left\|\left(I-K_{2} B^{-1}\right)^{-1}\right\|\|B\|^{3}\left\|\left(I-P_{n}\right) B^{-1} K_{2}\right\|<1,
$$

then $\left(I_{n}-K_{n}\right)$ is nonsingular and satisfies

$$
\left\|\left(I_{n}-K_{n}\right)^{-1}\right\|_{E_{n}} \leqslant \frac{\|B\|\left\|\left(I-K_{2} B^{-1}\right)^{-1}\right\|}{1-\delta_{n}} .
$$

Proof. The term

$$
\begin{aligned}
\left\|\left(1-p_{n} r_{n}\right) K_{2} B^{-1}\right\| & \leqslant\|B\|^{2}\left\|\left(I-P_{n}\right) B^{-1} K_{2} B^{-1}\right\| \\
& \leqslant\|B\|^{2}\left\|\left(I-P_{n}\right) B^{-1} K_{2}\right\|,
\end{aligned}
$$

since $\left\|B^{-1}\right\| \leqslant 1$ by Lemma 3.1(iii). 
The theorem follows directly from [14, Theorem 3.2]. We can now calculate the error $\left\|u-q_{n} \mathbf{v}_{n}\right\|$.

THEOREM 3.7. The error in Galerkin's method for the equation (3.1) satisfies

$$
\left\|u-q_{n} \mathbf{v}_{n}\right\| \leqslant\|B\|(1+C(n))\left\|\left(I-P_{n}\right) u\right\|
$$

where

$$
C(n)=\frac{\|B\|^{2}\left\|\left(I-K_{2} B^{-1}\right)^{-1}\right\|\left\|\left(I-Q_{n}^{*}\right) B^{*-1} K_{2}^{*}\right\|}{1-\delta_{n}},
$$

$Q_{n}=p_{n} r_{n}, \delta_{n}<1$ is in Eq. (3.16), and * denotes the adjoint.

Proof. Since $B u=v$, we have

$$
\begin{aligned}
\left\|u-q_{n} \mathbf{v}_{n}\right\| & \leqslant\left\|u-q_{n} B_{n}^{-1} s_{n} B u\right\|+\left\|q_{n}\left(B_{n}^{-1} s_{n} v-\mathbf{v}_{n}\right)\right\| \\
& =\left\|u-q_{n} B_{n}^{-1} s_{n} B u\right\|+\left\|r_{n} v-v_{n}\right\|_{E_{n}} .
\end{aligned}
$$

The first term is simply the error in Galerkin's method for the problem $B u=B u$, and

$$
\left\|u-q_{n} B_{n}^{-1} s_{n} B u\right\| \leqslant\|B\|\left\|\left(I-P_{n}\right) u\right\| .
$$

For the second term we use [14, Eq. 18] to get

$$
\left\|r_{n} v-\mathbf{v}_{n}\right\|_{E_{n}} \leqslant\left\|\left(I-K_{n}\right)^{-1}\right\|_{E_{n}}\left\|r_{n}\right\|\left\|K_{2} B^{-1}\left(1-p_{n} r_{n}\right) B u\right\| .
$$

A bound for $\left\|\left(I-K_{n}\right)^{-1}\right\|$ is given in Theorem 3.6 and

$$
\begin{aligned}
\| K_{2} B^{-1}(I & \left.-p_{n} r_{n}\right) B u \| \\
& =\left\|K_{2} B^{-1}\left(I-Q_{n}\right)^{2} B u\right\| \leqslant\left\|K_{2} B^{-1}\left(I-Q_{n}\right)\right\|\left\|\left(I-Q_{n}\right) B u\right\| \\
& \leqslant\left\|\left(I-Q_{n}^{*}\right) B^{*-1} K_{2}^{*}\right\|\|B\|^{2}\left\|\left(I-P_{n}\right) u\right\| .
\end{aligned}
$$

Hence,

$$
\begin{aligned}
\left\|u-q_{n} \mathbf{v}_{n}\right\| & <\|B\|\left(1+\frac{\|B\|^{2}\left\|\left(I-K_{2} B^{-1}\right)\right\|\left\|\left(I-Q_{n}^{*}\right) B^{*-1} K_{2}^{*}\right\|}{1-\delta_{n}}\right)\left\|\left(I-P_{n}\right) u\right\| \\
& =\|B\|(1+C(n))\left\|\left(I-P_{n}\right) u\right\| \quad \text { as required. }
\end{aligned}
$$

To discuss convergence as $n \rightarrow \infty$, we assume that $P_{n} g \rightarrow g$ as $n \rightarrow \infty$ for all $g \in L^{2}[-1,1]$. We can then produce

LEMMA 3.8. Suppose $P_{n} g \rightarrow g$ as $n \rightarrow \infty$ for all $g \in L^{2}[-1,1]$. Then

(i) $p_{n} r_{n} g \rightarrow g$ for all $g \in L^{2}[-1,1]$,

(ii) $\left(p_{n} r_{n}\right)^{*} g \rightarrow g$ for all $g \in L^{2}[-1,1]$.

(iii) $c(n) \rightarrow 0$ in (3.17).

Proof. (i) $B^{-1} g \in L^{2}[-1,1]$, so we can use Lemma 3.5.

(ii) Since $\left(p_{n} r_{n}\right)^{*} g=q_{n}\left(B^{*}\right)^{-1} s_{n} B^{*} g$, the convergence of $\left(p_{n} r_{n}\right)^{*} g$ to $g$ depends on the convergence of Galerkin's method for the problem $B^{*} w=B^{*} g$. It is readily verified that $B$ may be replaced by $B^{*}$ in the analysis prior to Theorem 3.2 , and the desired convergence is readily established. 
(iii) Since $\left(B^{*}\right)^{-1}$ is continuous and $K_{2}$ compact, the composition $\left(B^{*}\right)^{-1} K_{2}$ is compact and $\left\|\left(I-Q_{n}^{*}\right)\left(B^{*}\right)^{-1} K_{2}\right\| \rightarrow 0$ from a standard theorem in functional analysis; cf. [14]. Thus $c(n) \rightarrow 0$.

This lemma gives the interesting result

$$
\left\|\left(I-P_{n}\right) u\right\| \leqslant\left\|u-q_{n} \mathbf{v}_{n}\right\| \leqslant\|B\|\left\|\left(I-P_{n}\right) u\right\|+o(1),
$$

which is of the form (1.2).

4. Spline Functions as a Basis for Galerkin's Method. The spline functions form a convenient basis for Galerkin's method. One can often calculate the resulting "stiffness" matrix analytically. However, we saw from Section 2 that the solution of the singular integral equations under discussion invariably possesses endpoint singularities. Hence there is a need to use splines defined on nonuniform meshes and to possibly incorporate suitable singular functions into the basis.

The principal contributions to the theory of splines on nonuniform meshes have been Rice [13], de Boor [1], Burchard [2], and Dodson [5]. All of these authors have shown that with careful knot selection one can achieve asymptotically optimal rates of convergence $\left(O\left(N^{-k}\right)\right.$ for splines of degree less than $\left.k\right)$. The difficulty in applying the theory is that the function we wish to approximate is unknown. In this case we may use the known asymptotic behavior at the endpoints to calculate a good set of knots.

First we will define our notation. $\pi$ will denote a partition

$$
\pi: a=t_{0}<t_{1}<\cdots<t_{N}=b
$$

of the interval $[a, b] . P_{\pi}^{k}$ denotes the set of piecewise polynomials of degree less than $k$ and having breakpoints at the $t_{i}, i=1, \ldots, N-1$. We will find approximations in the class

$$
S_{\pi}^{k}= \begin{cases}P_{\pi}^{k}, & k=1, \\ P_{\pi}^{k} \cap C[a, b], & k \geqslant 2,\end{cases}
$$

and determine a partition to give $O\left(N^{-k}\right)$ rates of convergence in the space $L^{2}[a, b]$. (One can easily adapt the method to give estimates in more general spaces $L^{p}[a, b]$.)

The first lemma is adapted from [2, Lemma 1].

Lemma 4.1. Let $f \in C^{(k)}[a, b]$. Then there exist $s \in S_{k}^{\pi}$ and a constant $A_{k}$, independent of $f$, for which

(a)

$$
\max _{t_{i}<t<t_{i+1}}|f(t)-s(t)| \leqslant A_{k} h_{i}^{k} \max _{t_{i}<t<t_{i+1}}\left|f^{(k)}\right|
$$

for $i=0, \ldots, N-1$, and $h_{i}=t_{i+1}-t_{i}$;

(b) if $k>1$,

$$
s\left(t_{i}\right)=f\left(t_{i}\right), \quad i=0, \ldots, N .
$$

Proof. One can construct $s$ by equidistant Lagrange interpolation of degree $k-1$ in the interval $\left[t_{i}, t_{i+1}\right]$. Then $A_{k}=1 / k$ !. One takes $A_{1}=\frac{1}{2}$.

Remark. By a more judicious choice of interpolation points in $\left[t_{i}, t_{i+1}\right]$, one could obtain smaller values for $A_{k}$ when $k>2$. We can now produce our main theorem. 
THEOREM 4.2. Let $f \in C^{(k)}[a, b]$ and $g(t)$ be a continuous function that satisfies (i) $g(t) \geqslant \beta>0$, for $t \in[a, b]$,

(ii) $\left|f^{(k)}(t)\right|^{\sigma} \leqslant g(t)$, for $t \in[a, b]$ and $\sigma=2 /(2 k+1)$.

For an integer $N$, define the partition $\pi^{*}=\left(t_{i}\right)_{i=0}^{N}$ of $[a, b]$, where $t_{0}=a$ and

$$
\int_{t_{i}}^{t_{i+1}} g(t) d t=\frac{1}{N} \int_{a}^{b} g(t) d t .
$$

Then there exists an integer $N_{0}$ such that, for $N \geqslant N_{0}$, there exists $s^{*} \in S_{\pi^{*}}^{k}$ such that

$$
\left\|f-s^{*}\right\| \leqslant \frac{C_{k}}{N^{k}}\left\{\int_{a}^{b} g(t) d t\right\}^{k+1 / 2}
$$

where $C_{k}$ is independent of $N$ and $f$.

Proof (cf. [2]). $\log g(t)$ is uniformly continuous, so there exists $\delta>0$ such that

$$
\max _{x<t<x+\delta} g(t) \leqslant 2 \min _{x<t<x+\delta} g(t) .
$$

Also, for $t_{i}$ defined by (4.1),

$$
h_{i}=t_{i+1}-t_{i} \leqslant \frac{1}{\beta} \int_{t_{i}}^{t_{i+1}} g(t) d t=\frac{1}{N \beta} \int_{a}^{b} g(t) d t .
$$

We choose $N_{0}$ such that $h_{i}<\delta, i=0, \ldots, N-1$, if $N \geqslant N_{0}$. Then, for $N \geqslant N_{0}$ and $t \in\left[t_{i}, t_{i+1}\right]$,

$$
\begin{aligned}
\left|f^{(k)}(t)\right|^{\sigma} & \leqslant \max _{t_{i}<t<t_{i+1}} g(t) \leqslant 2 \min _{t_{i}<t<t_{i+1}} g(t) \\
& \leqslant \frac{2}{h_{i}} \int_{t_{i}}^{t_{i+1}} g(t) d t=\frac{2}{h_{i} N} \int_{a}^{b} g(t) d t .
\end{aligned}
$$

Hence, using the fact $\sigma=2 /(2 k+1)$,

$$
\left|f^{(k)}(t)\right|^{2} \leqslant\left(\frac{2}{h_{i} N} \int_{a}^{b} g(t) d t\right)^{2 k+1} \text { for } t \in\left[t_{i}, t_{i+1}\right] .
$$

Choose $s^{*} \in S_{\pi^{*}}^{k}$ from Lemma 1. Then

$$
\begin{aligned}
\left\|f-s^{*}\right\|^{2} & \leqslant \sum_{i=0}^{N-1} A_{k}^{2} h_{i}^{2 k+1} \max _{t_{i}<t<t_{i+1}}\left|f^{(k)}(t)\right|^{2} \\
& \leqslant \sum_{i=0}^{N-1} A_{k}^{2} h_{i}^{2 k+1}\left(\frac{2}{h_{i} N} \int_{a}^{b} g(t) d t\right)^{2 k+1}=\frac{A_{k}^{22^{2 k+1}}}{N^{2 k}}\left(\int_{a}^{b} g(t) d t\right)^{2 k+1} .
\end{aligned}
$$

Hence,

$$
\left\|f-s^{*}\right\| \leqslant \frac{C_{k}}{N^{k}}\left\{\int_{a}^{b} g(t) d t\right\}^{k+1 / 2},
$$

where $C_{k}=2^{k+1 / 2} A_{k}$.

The partition $\pi^{*}$ will produce a fine mesh in regions where $g(t)$ is large. However, the theorem is not directly applicable to functions with endpoint singularities. Dodson [5] has shown that a similar result will hold for functions whose $k$ th derivative has a finite number of singularities and is monotonic in a neighborhood of such singularities. The key property is the integrability of the function $g(t)$. It is instructive to consider the function $f(t)=t^{\alpha}\left(\alpha>-\frac{1}{2}\right)$ over the integral $[0,1]$. 
Here we would take

$$
g(t)=P_{\alpha, k} t^{2(\alpha-k) /(2 k+1)},
$$

where $P_{\alpha, k}$ is a constant. $g(t)$ is clearly integrable, and from (4.1) we recover the partition due to Rice [13]

$$
\begin{aligned}
t_{i} & =\left(\frac{i}{N}\right)^{q}, \quad i=0, \ldots, N \\
q & =\frac{2 k+1}{2 \alpha+1}
\end{aligned}
$$

This result is useful since it gives insight into how the knots should be placed near the endpoints. It also points out a practical difficulty. If $\alpha$ is close to $-\frac{1}{2}$, then $q$ will get very large, so, although in theory we can get $O\left(N^{-k}\right)$ rates of convergence, the distance between knots becomes so small that computing with them is well-nigh impossible. Also, one can calculate

$$
\left(\int_{0}^{1} g(t) d t\right)^{1 / \sigma}=\left(P_{\alpha, k} \frac{2 k+1}{2 \alpha+1}\right)^{k+1 / 2}
$$

and see that the size of this term will be large when $\alpha \sim-\frac{1}{2}$, and the accuracy obtained with this partition may only be modest.

The introduction of singular functions into the basis reduces, to a large extent, the problem of small intervals. We know that the solution of (1.1) has the form

$$
u(s)=(1-s)^{\alpha}(1+s)^{\beta} w(s),
$$

where $w(s)$ is smooth and $\alpha, \beta$ are known. For a given integer $N$, we construct a solution as follows. In an interval $\left[-1,-1+\delta_{1}(N)\right]$, where $\delta_{1}(N)$ is a function of $N$, we find an approximation of the form

$$
U_{N}(s)=C_{1}(1+s)^{\beta},
$$

and in an interval [1 $\left.-\delta_{2}(N), 1\right]\left(\delta_{2}(N)\right.$ is a function of $\left.N\right)$ we find an approximation of the form

$$
U_{N}(s)=C_{2}(1-s)^{\alpha} \text {. }
$$

$C_{1}, C_{2}$ are constants to be determined. $\delta_{1}(N)$ and $\delta_{2}(N)$ are chosen so that

$$
\begin{aligned}
\int_{-1}^{-1+\delta_{1}(N)}\left(u(s)-u_{N}(s)\right)^{2} d s & =O\left(\frac{1}{N^{2 k}}\right), \\
\int_{1-\delta_{2}(N)}^{1}\left(u(s)-u_{N}(s)\right)^{2} d s & =O\left(\frac{1}{N^{2 k}}\right) .
\end{aligned}
$$

In the interval $\left[-1+\delta_{1}(N), 1-\delta_{2}(N)\right], u(s)$ is to be approximated by a spline $u_{N}(s)$ of degree $k$ and with $N$ knots chosen according to Theorem 4.2.

If $\pi$ denotes a partition of $\left[-1+\delta_{1}, 1-\delta_{2}\right]$, we let $\tilde{S}_{\pi}^{k}$ be the space formed by augmenting the spline space with the singular functions. We can construct a function $\tilde{s} \in \tilde{S}_{\pi}^{k}$ such that $\|\tilde{s}-u\|=O\left(N^{-k}\right)$, as follows.

In the interval $\left[-1,-1+\delta_{1}\right], u(s)$ has the form

$$
u(s)=(1+s)^{\beta} v(s)=(1+s)^{\beta}\left\{v(-1)+(1+s) v^{\prime}(\xi)\right\}
$$

where $v(s)$ is smooth and $\xi \in\left(-1,-1+\delta_{1}\right)$. Hence, if $u(s)$ is approximated by $v(-1)(1+s)^{\beta}$ in $\left[-1,-1+\delta_{1}\right]$, the $L^{2}$ error will be 


$$
\left[\int_{-1}^{-1+\delta_{1}}(1+s)^{2 \beta+2}\left|v^{\prime}(\xi)\right|^{2} d s\right]^{1 / 2} \leqslant \frac{\delta_{1}^{\beta+1.5}}{\sqrt{2 \beta+3}} \max _{-1<s<-1+\delta_{1}}\left|v^{\prime}(s)\right| .
$$

Hence, by taking

$$
\delta_{1}^{\beta+1.5} / \sqrt{2 \beta+3}=N^{-k}
$$

i.e.

$$
\delta_{1}=(2 \beta+3)^{1 /(2 \beta+3)} N^{-k /(\beta+1.5)},
$$

we will achieve the desired rate of convergence. Similarly, we take

$$
\delta_{2}=(2 \alpha+3)^{1 /(2 \alpha+3)} N^{-k /(\beta+1.5)}
$$

to achieve the desired rate of convergence in $\left[1-\delta_{2}, 1\right]$.

To calculate a partition of $\left[-1+\delta_{1}, 1-\delta_{2}\right]$, we must find a suitable function $g(s)$ for use in Theorem 4.2. One can show that

$$
u^{(k)}(s)=(1-s)^{\alpha-k}(1+s)^{\beta-k} W_{k}(s)
$$

where $W_{k}(s)$ is bounded, and we put

$$
g(s)= \begin{cases}D(1-s)^{(\alpha-k) \sigma}, & 0<s<1, \\ D(1+s)^{(\beta-k) \sigma}, & -1<s<0,\end{cases}
$$

where $D$ is a constant chosen such that $g(s)>\left[u^{(k)}(s)\right]^{\sigma}$. It is then easy to show that

$$
\int_{-1+\delta_{1}}^{1-\delta_{2}} g(t) d t=D\left\{\frac{1}{\gamma_{1}}\left(1-\delta_{1}^{\gamma_{1}}\right)+\frac{1}{\gamma_{2}}\left(1-\delta_{2}^{\gamma_{2}}\right)\right\}
$$

with

$$
\gamma_{1}=(\beta-k) \sigma+1, \quad \gamma_{2}=(\alpha-k) \sigma+1 .
$$

The desired partition is calculated from (4.1) and is

$$
t_{i}=-1+\left\{\delta_{1}^{\gamma_{1}}+\frac{i}{N}\left(\left(1-\delta_{1}^{\gamma_{1}}\right)+\frac{\gamma_{1}}{\gamma_{2}}\left(1-\delta_{2}^{\gamma_{2}}\right)\right)\right\}^{1 / \gamma_{1}},
$$

for $i=0,1, \ldots, N^{*}$, and

$$
t_{i}=1-\left\{\delta_{2}^{\gamma_{2}}+\frac{(N-i)}{N}\left[\left(1-\delta_{2}^{\gamma_{2}}\right)+\frac{\gamma_{2}}{\gamma_{1}}\left(1-\delta_{1}^{\gamma_{1}}\right)\right]\right\}^{1 / \gamma_{2}},
$$

$$
i=N^{*}+1, \ldots, N
$$

where

$$
\frac{N^{*}}{N} \leqslant \frac{1-\delta_{1}^{\gamma_{1}}}{\left(1-\delta_{1}^{\gamma_{1}}\right)+\frac{\gamma_{1}}{\gamma_{2}}\left(1-\delta_{2}^{\gamma_{2}}\right)} .
$$

The integer $N^{*}$ is needed because of the different forms of $g(s)$ for $s$ negative and positive. The constant $D$ cancels out in the computation.

Hence we may show the existence of a function $\tilde{s} \in \tilde{S}_{\pi^{*}}^{k}$ with the desired rate of convergence. From Theorem 3.7 it will then follow that the rate of convergence of the Galerkin approximation $U_{N}(s)$, when our trial space is $\tilde{S}_{\pi^{*}}^{k}$, satisfies

$$
\left\|u-U_{N}\right\|=O\left(N^{-k}\right) \text {. }
$$


The numerical solution will suffer a mild discontinuity at the points $-1+\delta_{1}$, $1-\delta_{2}$, but numerical experience has shown that this is not a serious defect.

5. Numerical Example. Galerkin's method, using a basis of piecewise linear functions and the singular functions of Section 4, was implemented and applied to the example

$$
u(s)-\frac{1}{\pi} \int_{-1}^{+1} \frac{u(t)}{t-s} d t=1
$$

which has the exact solution

$$
u(s)=\frac{1}{\sqrt{2}}\left(\frac{1-s}{1+s}\right)^{1 / 4} .
$$

For $\lambda=1$, the following results were obtained. The first table gives the value of the constants $C_{1}, C_{2}$ of (4.2) and (4.3), together with $\delta_{1}$ and $\delta_{2}$ from (4.4) and (4.5).

\begin{tabular}{c|c|c|c|c}
$N$ & $C_{1}$ & $\delta_{1}$ & $C_{2}$ & $\delta_{2}$ \\
\hline 16 & 0.8405 & 0.0155 & 0.5971 & 0.0561 \\
32 & 0.8407 & 0.0054 & 0.5958 & 0.0263
\end{tabular}

The value of $C_{1}$ should tend to $2^{-1 / 4}=0.8409$ as $N \rightarrow \infty$, and $C_{2}$ should tend to $2^{-3 / 4}=0.5946$ as $N \rightarrow \infty$. We will now compare the Galerkin approximation with the true solution at interior points

\begin{tabular}{r|c|c|c}
$s$ & $N=16$ & $N=32$ & true \\
\hline-0.8 & 1.2256 & 1.2243 & 1.2247 \\
-0.4 & 0.8747 & 0.8738 & 0.8739 \\
0.0 & 0.7068 & 0.7073 & 0.7071 \\
0.4 & 0.5720 & 0.5721 & 0.5721 \\
0.8 & 0.4082 & 0.4082 & 0.4082
\end{tabular}

Faculty of Mathematical Studies

The University

Southampton SO9 5NH, England

1. C. DE BOOR, "Good approximation by splines with variable knots," Spline Functions and Approximation Theory (Proc. Sympos. Univ. of Alberta, 1972 (A. Meir and A. Sharma, Eds.)), Birkhauser, Basel, 1973, pp. 57-72.

2. H. G. Burchard, "Splines (with optimal knots) are better," Applicable Anal., v. 3, 1973/1974, pp. 309-319.

3. T. Carleman, "Sur la résolution de certaines équations intégrales," Ark. Mat. Astronom. Fys., v. 16, 1921 , pp. 1-19.

4. Y. Cherruault, Approximation d'Opérateurs Linéaires et Applications, Dunod, Paris, 1968.

5. D. S. Dodson, Optimal Order Approximation by Polynomial Spline Functions, Ph. D. Thesis, Comp. Sci. Dept., Purdue Univ., Lafayette, Ind., 1972.

6. M. L. Dow \& D. Elliort, "The numerical solution of singular integral equations over $(-1,1)$," SIAM J. Numer. Anal., v. 16, 1979, pp. 115-134.

7. F. ERdogan, G. D. GuPTA \& T. S. CoOK, "Numerical solution of singular integral equations," Mech. Fract., v. 1, 1973, pp. 368-425.

8. V. V. Ivanov, The Theory of Approximate Methods and Their Applications to the Numerical Solution of Singular Integral Equations, Noordhoff, Leyden, 1976. 
9. L. N. KARPJENKo, "Approximate solution of singular integral equations by means of Jacobi polynomials,” Prikl. Mat. Meh., v. 30, 1966, pp. 564-569; English transl., J. Appl. Math. Mech., v. 30, 1967, pp. 668-675.

10. S. KRENK, "A quadrature formula for singular integral equations of the first and second kind," Quart. Appl. Math., v. 33, 1975, pp. 225-232.

11. P. LiNZ, "A general theory for the approximate solution of operator equations of the second kind," SIAM J. Numer. Anal., v. 14, 1977, pp. 543-553.

12. N. I. MUSHKHELISHVILI, Singular Integral Equations, Noordhoff, Groningen, 1953.

13. J. R. RICE, "On the degree of convergence of nonlinear spline approximation," Approximation with Special Emphasis on Spline Functions (I. J. Schoenberg, Ed.), Academic Press, New York, 1969, pp. 349-365.

14. K. S. Thомas, "On the approximate solution of operator equations," Numer. Math., v. 23, 1975, pp. 231-239.

15. F. G. Tricomi, Integral Equations, Pure and Appl. Math., vol. 5, Interscience, New York, 1957. 